\title{
Contrast-enhanced ultrasonography: does it have a role in developing countries?
}

\author{
Richard M Mendelson ${ }^{1}$ \\ ${ }^{1}$ Emeritus Consultant Radiologist, Royal Perth Hospital, Western Australia \\ Clinical Professor, University of Western Australia and Notre Dame University, Western \\ Australia
}

\begin{abstract}
Contrast-enhanced ultrasonography (CEUS) has become an extremely useful adjunct to conventional ultrasound, and in many clinical situations it may replace or obviate the need for CT or MRI. Its use in developed countries has been variable, but it is suggested that in developing countries where CT and MRI have more limited availability, CEUS has great potential. This article explains why the author believes this to be the case, introduces the technique and describes the clinical roles to which CEUS may be applied.
\end{abstract}

Keywords:Ultrasonography; contrast media; microbubbles; diagnostic imaging; countries, developing

Copyright : Richard M Mendelson

Correspondence : Richard M Mendelson, Richard.Mendelson@health.wa.gov.au 


\section{Introduction}

Contrast-enhanced Ultrasonography (CEUS) has been in clinical use for many years, its most widespread application having been in cardiology. Advances in the contrast agents have lead to the ability of modern generation of the microbubbles to transit from peripheral veins, through the lung vasculature to the systemic circulation, and to recirculate for several minutes. This has resulted in the clinical roles to widen markedly. Only the non-cardiological roles will be discussed in this article.

CEUS has become an extremely useful adjunct to conventional ultrasound ${ }^{1,2}$ and, in many scenarios, may replace contrastenhanced CT or MRI, particularly in the examination of the solid viscera of the abdomen. Despite this, its uptake in developed countries has been variable. CEUS is widely used in parts of Europe. However, in Australia, for example, CEUS is essentially confined to major public health centres $^{3}$, partly due to the fee structure for medical procedures, partly due to the lack of incentive (and possibly the presence of a disincentive in private radiology practice) to the use of CEUS when CT and MRI are liberally available, and perhaps also partly due to the diminishing 'hands-on' experience of radiologists in US examinations. In the USA, while similar factors may apply, a major issue is that the recent generation of contrast agents has not been approved by the Food and Drugs Administration. The causes for this patchy uptake in the developed world was excellently summarized by Sidhu ${ }^{4}$ in 2008 . Since then the impression is that progress has been slow.

However, there is a huge untapped potential for CEUS in the developing world and this article discusses the advantages of CEUS in the context of the health system of those countries where access to CT and, in particular, MRI is limited. It is my understanding that in Sri Lanka, for example, outside the major population centres, CT and MRI are present in the minority of sites, whereas US is second only to plain radiography in availability.

When CEUS is a viable clinical alternative to $\mathrm{CT}$ and MRI, its advantages include:

1). Lack of need for transport of patients to major centers for CT/MRI

$2)$. Less cost to the health system:

o Less requirement for capital outlay on CT/MR machines, building infrastructure and staffing

- Less maintenance costs

- No requirement for strict oversight of ionizing radiation by regulation authorities

3). CEUS can be performed on existing modern US machines, uploaded with appropriate software (see below)

4). CEUS can be performed immediately at the same attendance as the conventional US

5). CEUS can be undertaken at the bedside, in the Emergency Room or clinic

6). The contrast agents are safe, even in the presence of renal impairment, with no need for preliminary laboratory testing.

7). The contrast is relatively cheap.

8). Lack of ionizing radiation ( $c f \mathrm{CT}$ )

9). The contrast medium is administered by peripheral intravenous injection.

\section{Ultrasound Contrast and Technique}

Latest generation US contrast agents consist 
of microbubbles of stable perfluorocarbon or sulfur hexafluoride gas in a tough shell of albumen or phospholipids. They are administered by peripheral intravenous injection and are predominantly blood pool agents - that is they remain within the intravascular space, unlike the majority of CT and MRI contrast agents. The encapsulated microbubbles measure $<10$ $\mu \mathrm{m}$ and thus pass through the pulmonary and systemic circulation and are durable enough to re-circulate for several minutes . The limited diffusion of the inert gas, as well as its low solubility, contribute to the persistence of the microspheres in the blood. The gas is inert, is not metabolised and is eliminated through the lungs within 10 minutes of administration.

US contrast agents work by increasing the strength of the backscattered signals from blood by several orders of magnitude. Imaging is acquired in real time as the agent enhances the vascular structures within normal and pathological structures. Effectively a 'real-time' US angiogram is obtained over several minutes, if required, during multiple vascular phases.
Contrast-specific scanning modes are required on the US machine ${ }^{5}$. These modes use a low mechanical index (MI) to avoid bursting the bubbles on insonation and are usually a variant of contrast harmonic imaging /phase inversion techniques. 'Flash' imaging can be used as an adjunct; this entails transiently increasing the MI to deliberately disrupt the microbubbles within the insonated area, and then returning to low MI to visualize contrast replenishment within the area of interest.

Images can be interpreted qualitatively (Figs $1-3$ ) and quantitatively (Fig 4). Quantitative assessment is achieved by prescribing regions of interest (ROI) in the image ${ }^{6}$. The software then plots Time versus Intensity curves (TICs) that it displays graphically. TICs are very useful to measure various objective parameters, such as time-to-peak intensity, maximum intensity, area-underthe-curve, wash-out, etc. This helps to characterise lesions, particularly when ROIs from a lesion (and even from different parts of a lesion) and surrounding normal tissue are compared.

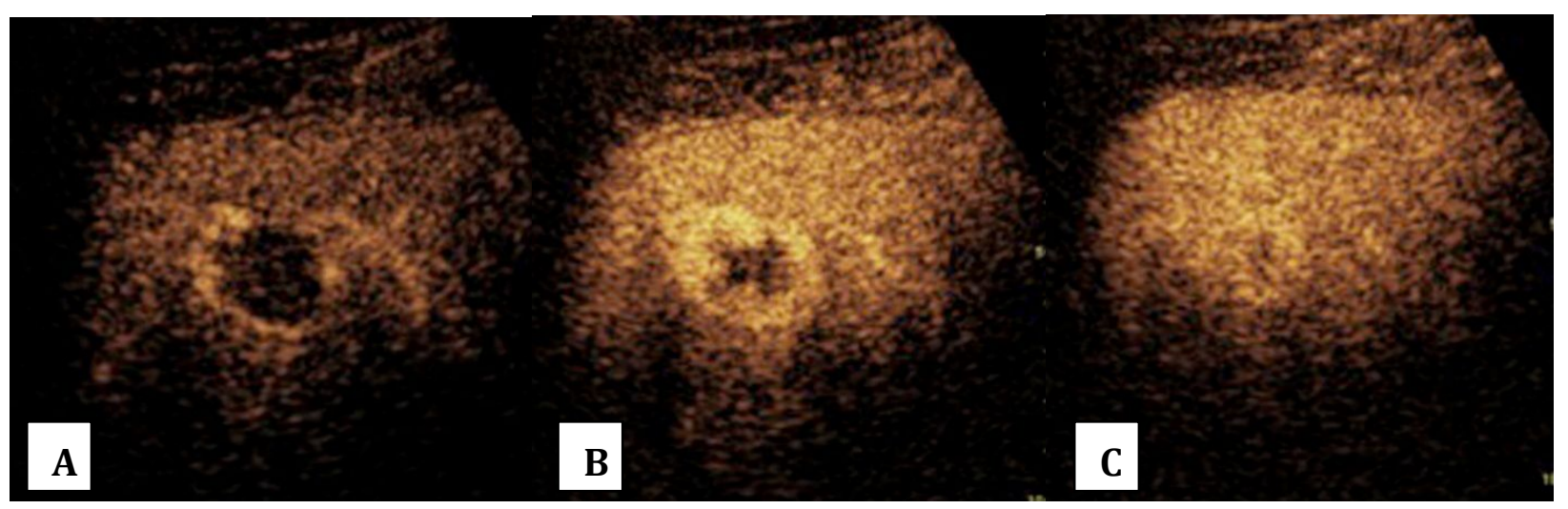

Fig.1 A,B,C._Serial images after IV US contrast agent of a liver haemangioma showing typical 'filling-in' of lesion from rim extending centrally. 


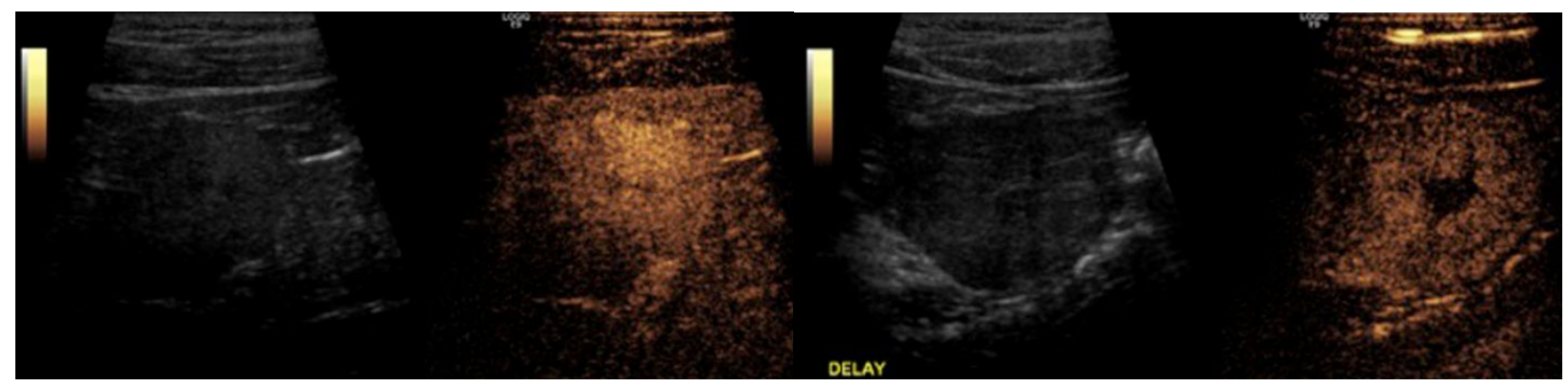

Fig.2 A,B._Serial images - early (A) and delayed (b) - after IV US contrast agent of hepatic Focal Nodular Hyperplasia, (FNH) showing early hyper-enhancement of the lesion with persistence of enhancement in (B). Note central 'scar' in (B)

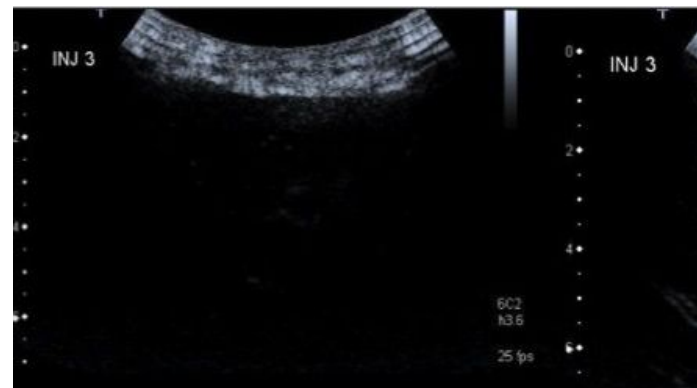

1. TIME 04:41:10 PM

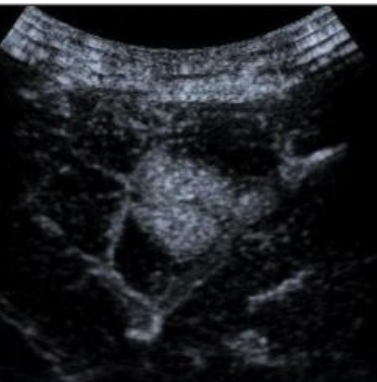

2. TIME 04:41:33 PM

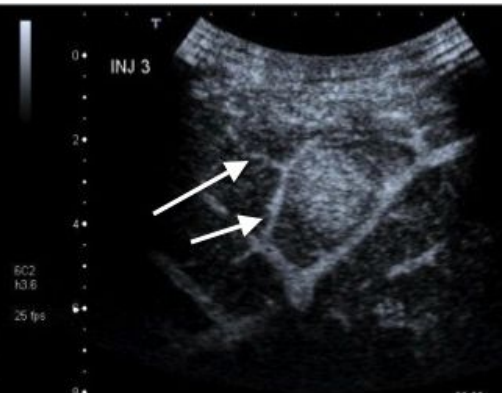

3. TIME 04:41:38 PM

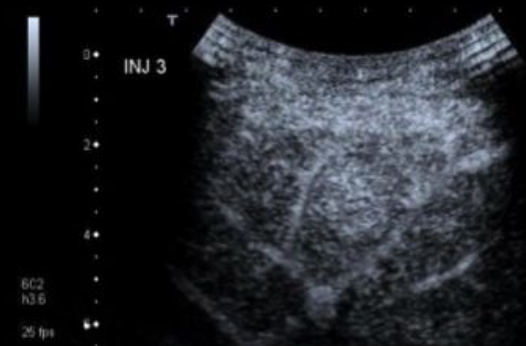

4. TIME 04:41:42 PM

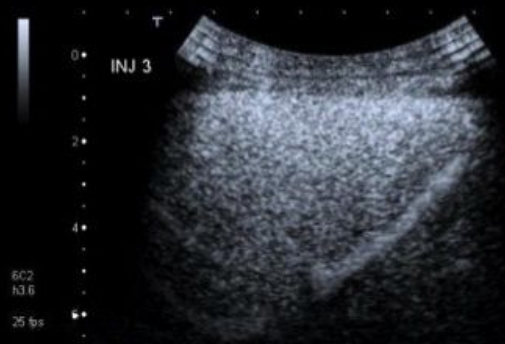

5. TIME 04:42:00 PM

Fig.3._Another patient with hepatic FNH, showing early (23 second) hyper-enhancement of the lesion becoming iso-echoic to liver at 50 seconds. Note prominent feeding vessels (arrowed)

\section{Complications and contra-indications}

US contrast agents are very safe. There is no requirement for laboratory tests of renal or thyroid function prior to administration. Hypersensitivity reactions are rare, with an incidence of life-threatening anaphylaxis reported as $0.001 \%{ }^{2}$. 


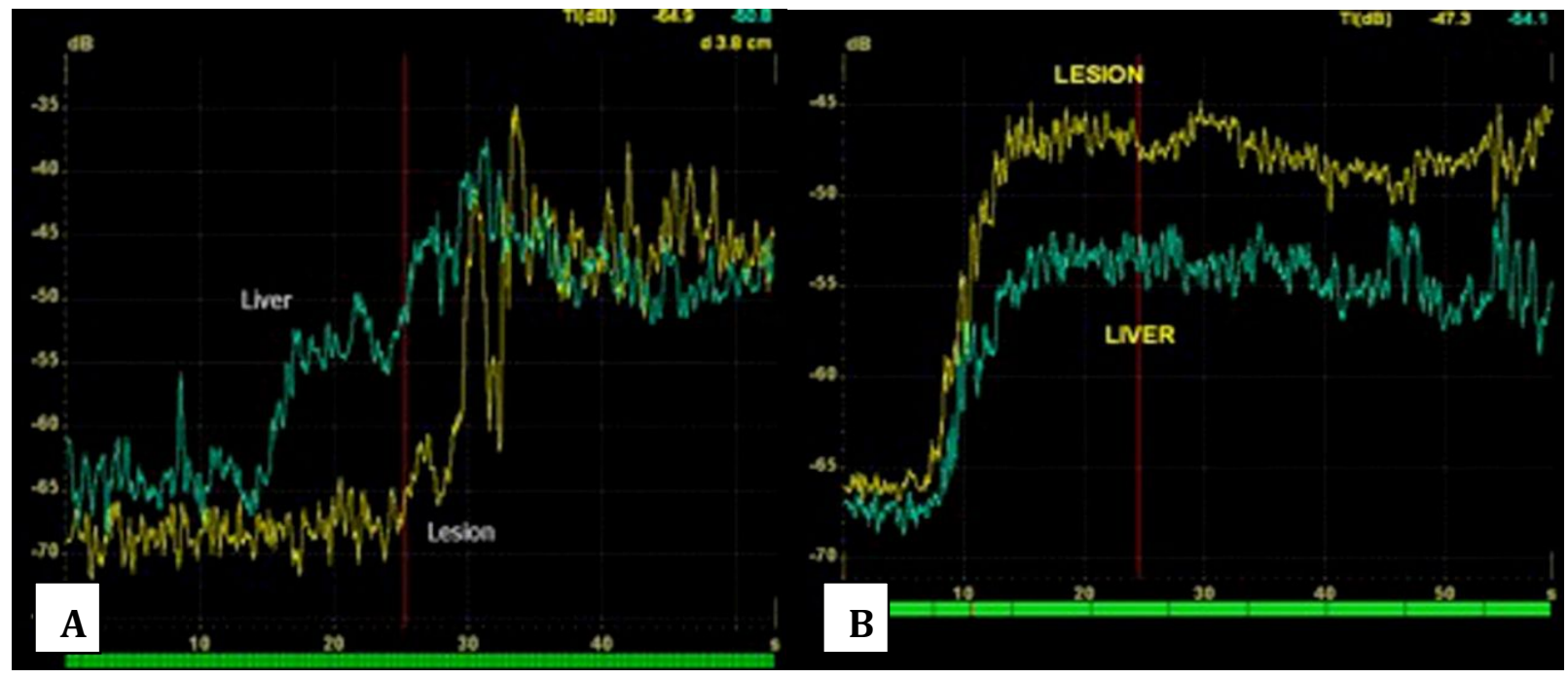

Fig.4 A, B._Same patients as Fig.1 and Fig.2, respectively. Time Intensity Curves (TICs) confirming graphically the enhancement of the lesion (yellow line) relative to normal liver (green line).

There is a theoretical risk that US with contrast agents could result in bioeffects. Experimental data from their use in small animals indicate that rupture of microvessels could occur when gas bodies are insonated. If this is a real risk it is likely only to be clinically significant when CEUS is used for ocular roles and when insonating brain without an intact skull 7 .

US contrast agents are not licensed in pregnancy. The question of use in paediatrics is complex; CEUS should provide a very beneficial modality in children in view of its lack of ionizing radiation. However, in many jurisdictions the agents are not yet registered for paediatric use. Further developments are awaited, but in the meantime clinical judgement must remain important. A potentially important role of CEUS is the investigation of vesico-ureteric reflux after the intracavitary instillation of the contrast agent into the bladder.
Side effects of US contrast agents are unusual and almost always mild. Headache is the most frequent followed by nausea and/or vomiting, a warm sensation or flushing, dizziness and back pain.

With regard to contraindications, it was previously considered that patients with right-to-left cardiac shunts should not be given US contrast agents. However, recent data suggest that this should no longer be considered to be the case in adults ${ }^{8,9}$.

Caution should be taken when considering CEUS in patients with pulmonary hypertension and "unstable cardiopulmonary conditions", although in cardiology patients requiring echocardiography, the benefits of using US contrast agents may over-ride the risks $^{10}$.

For the contrast agents in which the shell of the microbubble is albumin, a history of allergy to albumin or blood products should be sought as a contraindication. 


\section{Technical limitations}

EUS is subject to the limitations of conventional US, including unfavourable body habitus and interposition of gas or bone between the skin surface and the target lesion. In addition, due to the nature of the CEUS scanning modes, deep lesions may not be able to be adequately examined with CEUS.

\section{Clinical roles}

CEUS provides a good alternative to $\mathrm{CT}$ and MRI in the examination of many solid organs, especially in the abdomen, and of vascular structures. The enhancement patterns of lesions can be studied during multiple vascular phases -arterial, portal venous, late and post-vascular phases - in a similar fashion to contrast-enhanced CT and contrast-enhanced MRI, but in real time with a resultant much higher temporal resolution than is possible with CT or MRI. The real-time nature of CEUS allows depiction of early arterial phase enhancement, which is sometimes missed on $\mathrm{CT}$ and MRI because of their lower 'frame rates'. The following is a noncomprehensive list of the applications of CEUS in various organs and clinical scenarios.

\section{Liver $^{2}$}

1). CEUS has proven useful in the characterization of focal liver lesions (FLLs). It is particularly useful for differentiating benign and malignant nodules $^{11,12}$. CEUS shows tissue perfusion analogous to that shown on contrastenhanced CT and MRI in which patterns

of enhancement in the arterial and portal venous phases predict the diagnoses of
FLLs $^{2,13-16}$ and has been shown to be comparable to $\mathrm{CT}$ and $\mathrm{MRI}^{17}$ and useful as a first line investigation of $\mathrm{FLLs}^{15}$. Most malignant lesions show 'wash-out' of contrast agent on portal venous or later phases (Fig 5).

2). For metastasis detection in patients with non-hepatic primary tumours, CEUS is better than conventional ultrasound ${ }^{18}$, but probably less sensitive than multi-detector CT on a lesion-by-lesion analysis ${ }^{19,20}$

3). The use of CEUS is cost-efficient in the first-line diagnosis of FLLs compared to CT and MRI ${ }^{21}$. CEUS can often establish a definitive diagnosis or otherwise aid in deciding whether a liver lesion needs further investigation.

4). Simple algorithms allow diagnosis of most liver masses ${ }^{22}$.

5). CEUS has been found to improve the characterisation of focal liver lesions with enhancement patterns observed during the arterial, portal venous and late phases generally similar to CECT and CEMRI.

6). CEUS is valuable for the evaluation of nodules in the patient at risk for hepatocellular carcinoma ${ }^{23,24}$.

7). CEUS can differentiate between adenoma and focal nodular hyperplasia in asymptomatic young women ${ }^{25,26}$ (Fig 6).

\section{$\underline{\text { Renal applications }}$}

CEUS provides information on tissue perfusion and may play a role in kidney mass characterization similar to the role of contrast-enhanced $\mathrm{CT}$ and $\mathrm{MRI}^{1,27}$.

Specific roles in the kidney include:

1). Differentiation of solid and cystic lesions in hypovascular masses.

2).CEUS significantly improves diagnostic confidence for solid renal masses. 


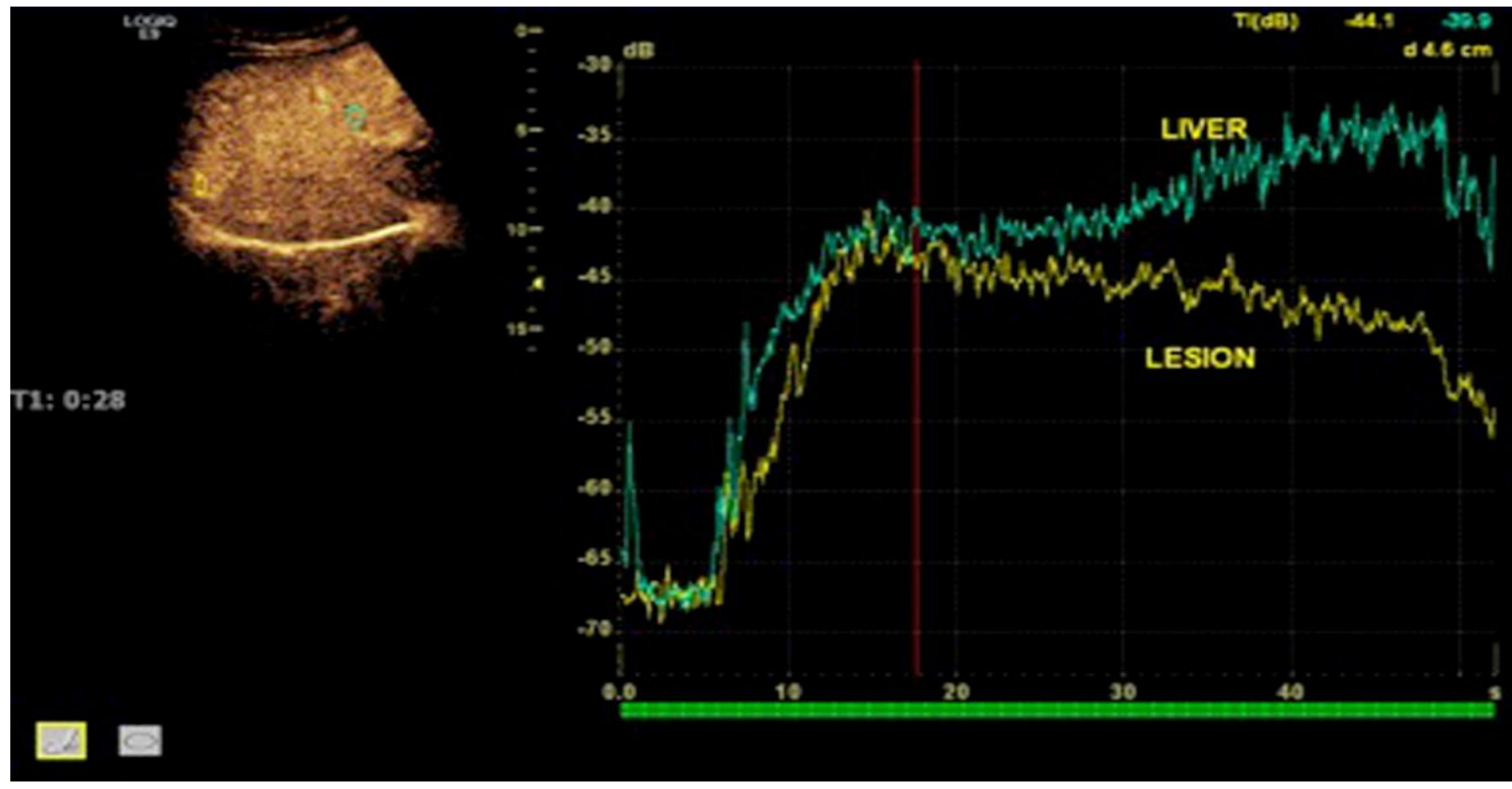

Fig.5. TIC of hepatocellular cancer. The TIC shows arterial enhancement of the lesion, similar to surrounding liver, followed by 'wash-out' of contrast from the lesion i.e. lesion becomes iso-echoic to liver

The overall accuracy for renal carcinoma has been shown to be $90 \%{ }^{28}$.

3). CEUS is useful for the diagnosis of renal pseudo-tumours, reducing the need for CT 29.

4). CEUS enables Bosniak classification of complex renal cysts without the need for $\mathrm{CT}^{30}$.

5). Investigation of suspected renal infarction - total or segmental

6). Vesico-ureteric reflux in girls after introduction of US contrast material into the bladder.

\section{$\underline{\text { Scrotal imaging }}$}

CEUS is useful in patients with acute scrotal pain $^{31}$ and trauma ${ }^{32}$.

1). In the differentiation of hypovascular and avascular lesions, the latter being indicative of benign disease
2). Discrimination of areas of non-viable tissue in cases of testicular trauma

3). Discrimination of abscess formation in severe epididymo-orchitis.

4). Detection of segmental infarction.

\section{$\underline{\text { Pancreas }}$}

CEUS has been shown to be effective in:

1). The characterisation, delineation and local staging of adenocarcinoma ${ }^{33}$.

2). The discrimination of mass-forming chronic pancreatitis and adenocarcinoma ${ }^{34}$.

3). The differentiation of cystic tumours from pseudocysts ${ }^{35}$.

4). Assessing hypervascular masses such as neuroendocrine tumours of the pancreas 36,37 . 


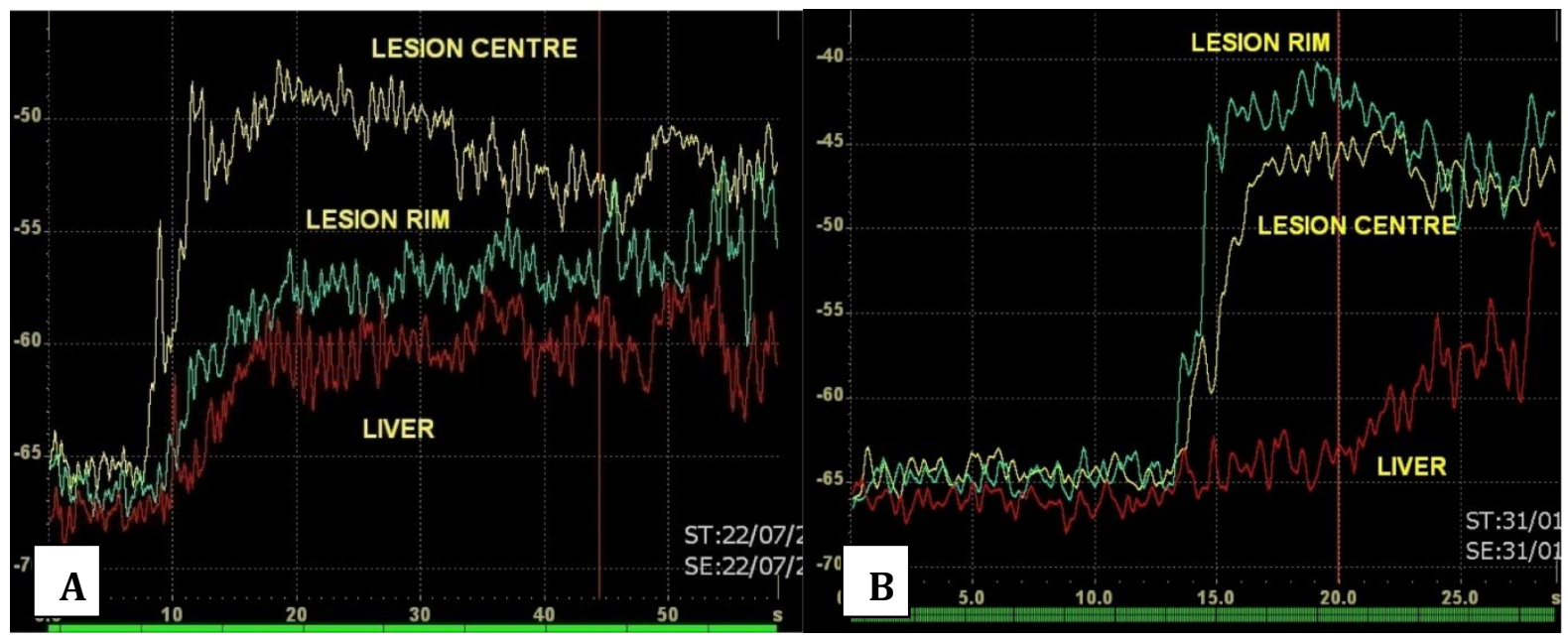

Fig.6 A,B. FNH (a) and adenoma (b). TICs from rim of lesion, central lesion and normal liver, showing different patterns of enhancement. FNH shows early enhancement of central portion followed by rim. Adenoma tends to show opposite pattern

\section{$\underline{\text { Trauma }}$}

CEUS has been shown to be an accurate technique for evaluating traumatic lesions of solid abdominal organs ${ }^{38,39}$. However, it has been suggested that its use should be as a first-line approach in patients with lowenergy isolated abdominal trauma, as it shows a high sensitivity both in lesion detection and grading, but that CT should be performed in CEUS-positive patients to exclude active bleeding and urinomas ${ }^{40}$. It is also useful for monitoring patients undergoing conservative treatment in mild degrees of liver and spleen trauma ${ }^{41}$.

\section{$\underline{\text { Non-cardiological vascular roles }}$}

CEUS may be used in a variety of vascular applications, including:

1). Improving US visualization of technically difficult vessels, such as the renal arteries in suspected renal artery stenosis $^{42}$.

2). Differentiation between severe stenosis and complete occlusion of major vessels, including visceral and cerebral arteries. In the carotid arteries CEUS improves the sensitivity of Doppler US and can be used for differentiating complete occlusion from residual flow in tight stenosis. CEUS can be also be utilised to improve the delineation of the inner vascular wall, enabling detection of the configuration of pre-stenotic, intrastenotic and post-stenotic segments and evaluation of neovascularization of plaque (which may be a precursor of plaque rupture $)^{43}$.

3). Assessment of vascular dissections

4). Evaluation of endoleaks following aortic 
aneurysm repair.

\section{$\underline{\text { Other applications }}$}

There are many other potential roles for CEUS, many of which await sufficient evidence of efficacy for clinical application. Examples include the investigation of gynaecological masses, assessment of renal transplants and intracavitary uses, such as voiding urosonography and imaging of Fallopian tube patency.

\section{Conclusion}

Contrast-enhanced ultrasonography is a procedure that holds great potential in the context of the health system of a developing country, where access to CT and MRI is limited. It is safe, relatively inexpensive, to a large extent can be applied with only relatively minor modification to existing resources, has a wide range of common clinical applications and may obviate the need in many patients for transfer to a major centre for CT or MRI.

\section{References}

1. Piscaglia, F., et al., The EFSUMB Guidelines and Recommendations on the Clinical Practice of Contrast Enhanced Ultrasound (CEUS): update 2011 on non-hepatic applications. Ultraschall Med, 2012. 33(1): p. 33-59.

2. Claudon, M., et al., Guidelines and good clinical practice recommendations for contrast enhanced ultrasound (CEUS) in the liver--update 2012: a WFUMBEFSUMB initiative in cooperation with representatives of AFSUMB, AIUM, ASUM, FLAUS and ICUS. Ultraschall Med, 2013. 34(1): p. 11-29.
3. Won, S.Y., et al., Hepatic contrastenhanced ultrasound: impact of its introduction in the Australian context. J Med Imaging Radiat Oncol, 2014. 58(1): p. $38-45$.

4. Sidhu, P.S., The EFSUMB guidelines for contrast-enhanced ultrasound are comprehensive and informative for good clinical practice: will radiologists take the lead? Br J Radiol, 2008. 81(967): p. 524-5.

5. Quaia, E., Microbubble ultrasound contrast agents: an update. Eur Radiol, 2007. 17(8): p. 1995-2008.

6. Salvatore, V., et al., Quantification of enhancement of focal liver lesions during contrast-enhanced ultrasound (CEUS). Analysis of ten selected frames is more simple but as reliable as the analysis of the entire loop for most parameters. Eur J Radiol, 2012. 81(4): p. 709-13.

7. Blomley, M., M. Claudon, and D. Cosgrove, WFUMB Safety Symposium on Ultrasound Contrast Agents: clinical applications and safety concerns. Ultrasound Med Biol, 2007. 33(2): p. 180-6.

8. Kalra, A., G.R. Shroff, and C.A. Herzog, Safety of ultrasound contrast agents in patients with intracardiac shunts. J Am Soc Echocardiogr, 2014. 27(12): p. 1359.

9. Parker, J.M., et al., Safety of ultrasound contrast agents in patients with known or suspected cardiac shunts. Am J Cardiol, 2013. 112(7): p. 1039-45.

10. Main, M.L., Ultrasound contrast agent safety: from anecdote to evidence. JACC Cardiovasc Imaging, 2009. 2(9): p. 1057-9.

11. Wilson, S.R. and P.N. Burns, An algorithm for the diagnosis of focal liver 
masses using microbubble contrastenhanced pulse-inversion sonography. AJR Am J Roentgenol, 2006. 186(5): p. 1401-12.

12. Sporea, I., et al., Contrast-enhanced ultrasound (CEUS) for the evaluation of focal liver lesions - a prospective multicenter study of its usefulness in clinical practice. Ultraschall Med, 2014. 35(3): p. 259-66.

13. Seitz, K., et al., Contrast-enhanced ultrasound (CEUS) for the characterization of focal liver lesions in clinical practice (DEGUM Multicenter Trial): CEUS vs. MRI--a prospective comparison in 269 patients. Ultraschall Med, 2010. 31(5): p. 492-9.

14. Seitz, K., et al., Contrast-Enhanced Ultrasound (CEUS) for the characterization of focal liver lesions prospective comparison in clinical practice: CEUS vs. CT (DEGUM multicenter trial). Parts of this manuscript were presented at the Ultrasound Dreilandertreffen 2008, Davos. Ultraschall Med, 2009. 30(4): p. 383-9.

15. Sporea, I., et al., Characterization of focal liver lesions using contrast enhanced ultrasound as a first line method: a large monocentric experience. J Gastrointestin Liver Dis, 2014. 23(1): p. 57-63.

16. Sporea, I., et al., Contrast enhanced ultrasound for the characterization of focal liver lesions. Med Ultrason, 2011. 13(1): p. 38-44.

17. Ryu, S.W., et al., Clinically useful diagnostic tool of contrast enhanced ultrasonography for focal liver masses: comparison to computed tomography and magnetic resonance imaging. Gut Liver, 2014. 8(3): p. 292-7.
18. Piscaglia, F., et al., Real time contrast enhanced ultrasonography in detection of liver metastases from gastrointestinal cancer. BMC Cancer, 2007. 7: p. 171.

19. Larsen, L.P., et al., Can contrastenhanced ultrasonography replace multidetector-computed tomography in the detection of liver metastases from colorectal cancer? Eur J Radiol, 2009. 69(2): p. 308-13.

20. Rojas Llimpe, F.L., et al., Imaging in resectable colorectal liver metastasis patients with or without preoperative chemotherapy: results of the PROMETEO-01 study. Br J Cancer, 2014. 111(4): p. 667-73.

21. Sirli, R., et al., Contrast enhanced ultrasound in focal liver lesions--a cost efficiency study. Med Ultrason, 2010. 12(4): p. 280-5.

22. Wilson, S.R., L.D. Greenbaum, and B.B. Goldberg,Contrast-enhanced ultrasound: what is the evidence and what are the obstacles? AJR Am J Roentgenol, 2009. 193(1): p. 55-60.

23. Zheng, S.G., H.X. Xu, and L.N. Liu, Management of hepatocellular carcinoma: The role of contrastenhanced ultrasound. World J Radiol, 2014. 6(1): p. 7-14.

24. Tanaka, H., et al., Cost-effectiveness analysis on the surveillance for hepatocellular carcinoma in liver cirrhosis patients using contrastenhanced ultrasonography. Hepatol Res, 2012.

25. Kim, T.K., et al., Focal nodular hyperplasia and hepatic adenoma: differentiation with low-mechanicalindex contrast-enhanced sonography. 
AJR Am J Roentgenol, 2008. 190(1): p. 58-66.

26. Bartolotta, T.V., et al., Hepatic focal nodular hyperplasia: contrast-enhanced ultrasound findings with emphasis on lesion size, depth and liver echogenicity. Eur Radiol, 2010. 20(9): p. 2248-56.

27. Siracusano, S., et al., The current role of contrast-enhanced ultrasound (CEUS) imaging in the evaluation of renal pathology. World J Urol, 2011. 29(5): p. 633-8.

28. Zhou, X., et al., Characterization and diagnostic confidence of contrastenhanced ultrasound for solid renal tumors. Ultrasound Med Biol, 2011. 37(6): p. 845-53.

29. Mazziotti, S., et al., Usefulness of contrast-enhanced ultrasonography in the diagnosis of renal pseudotumors. Abdom Imaging, 2010. 35(2): p. 241-5.

30. Ascenti, G., et al., Complex cystic renal masses: characterization with contrastenhanced US. Radiology, 2007. 243(1): p. $158-65$.

31. Valentino, M., et al., Role of contrast enhanced ultrasound in acute scrotal diseases. Eur Radiol, 2011. 21(9): p. 1831-40.

32. Hedayati, V., et al., Contrast-enhanced ultrasound in testicular trauma: role in directing exploration, debridement and organ salvage. $\mathrm{Br} \mathrm{J}$ Radiol, 2012. 85(1011): p. e65-8.

33. D'Onofrio, M., et al., Contrast-enhanced ultrasound of the pancreas. World $\mathrm{J}$ Radiol, 2010. 2(3): p. 97-102.
34. D'Onofrio, M., et al., Mass-forming pancreatitis: value of contrast-enhanced ultrasonography. World J Gastroenterol, 2006. 12(26): p. 4181-4.

35. D'Onofrio, M., et al., Comparison of contrast-enhanced sonography and MRI in displaying anatomic features of cystic pancreatic masses. AJR Am J Roentgenol, 2007. 189(6): p. 1435-42.

36. D'Onofrio, M., et al., Neuroendocrine pancreatic tumor: value of contrast enhanced ultrasonography. Abdom Imaging, 2004. 29(2): p. 246-58.

37. Dietrich, C.F., et al., Improved characterisation of solitary solid pancreatic tumours using contrast enhanced transabdominal ultrasound. J Cancer Res Clin Oncol, 2008. 134(6): p. 635-43.

38. Valentino, M., et al., Contrast-enhanced ultrasonography in blunt abdominal trauma: considerations after 5 years of experience. Radiol Med, 2009. 114(7): p. 1080-93.

39. Catalano, O., et al., CEUS in abdominal trauma: multi-center study. Abdom Imaging, 2009. 34(2): p. 225-34.

40. Sessa, B., et al., Blunt abdominal trauma: role of contrast-enhanced ultrasound (CEUS) in the detection and staging of abdominal traumatic lesions compared to US and CE-MDCT. Radiol Med, 2014.

41. Manetta, R., et al., Ultrasound enhanced with sulphur-hexafluoride-filled micro bubbles agent (SonoVue) in the follow- 
up of mild liver and spleen trauma. Radiol Med, 2009. 114(5): p. 771-9.

42. Blebea, J., et al., Duplex imaging of the renal arteries with contrast enhancement. Vasc Endovascular Surg, 2003. 37(6): p. 429-36.

43. Clevert, D.A., et al., The role of contrast-enhanced ultrasound in imaging carotid arterial diseases. Semin Ultrasound CT MR, 2013. 34(3): p. 20412. 